\title{
Some like it hot: A differential response to changing temperatures by the malaria vectors Anopheles funestus and An. gambiae s.l.
}

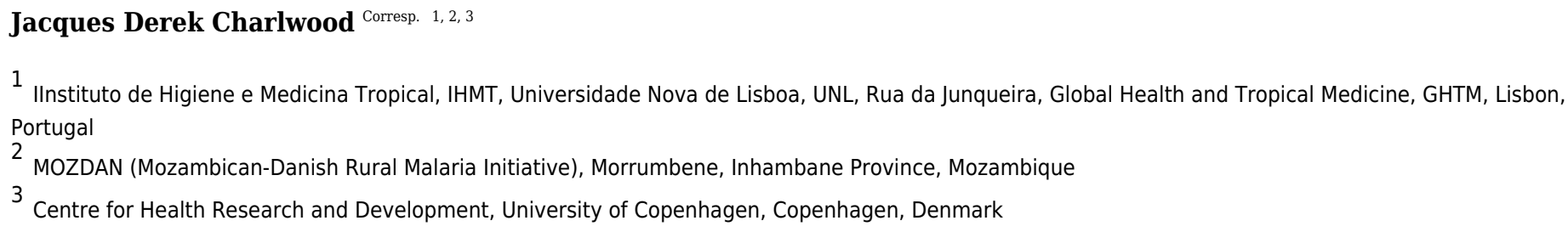

Background: With the possible implications of global warming, the effect of temperature on the dynamics of malaria vectors in Africa has become a subject of increasing interest. Information from the field is, however, relatively sparse. We describe the effect of ambient temperature over a five-year period on the dynamics of An. funestus and An. gambiae s.l., collected from a single village in southern Mozambique where temperatures varied from a night-time minimum of $6^{\circ} \mathrm{C}$ in the cool season to a daytime maximum of $35^{\circ} \mathrm{C}$ in the hot season. Results: Mean daily air temperatures varied from $34^{\circ} \mathrm{C}$ to $20^{\circ} \mathrm{C}$ and soil temperatures varied from $26^{\circ} \mathrm{C}$ to $12^{\circ} \mathrm{C}$. Diurnal variation was greatest in the cooler months of the year and were greater in air temperatures than soil temperatures. During the study 301, 705 female An. funestus were collected in 6043 light-trap collections, 161, 466 in 7397 exit collections and 16, 995 in 1315 resting collections. The equivalent numbers for An. gambiae s.I. are 72, 475 in light-traps, 33, 868 in exit collections and 5, 333 from indoor resting collections. Numbers of mosquito were greatest in the warmer months. Numbers of An. gambiae s.l. went through a one hundredfold change (from a mean of 0.14 mosquitoes a night to 14 ) whereas numbers of An. funestus merely doubled (from a mean of 20 to 40 a night). The highest environmental correlations and mosquito numbers were between mean air temperature $\left(r^{2}=0.52\right.$ for $A n$. funestus and 0.77 for $A n$. gambiae s.l.). Numbers of mosquito collected were not related to rainfall with lags of up to four weeks. Numbers of both gravid and unfed An. gambiae complex females in exit collections continued to increase at all temperatures recorded but gravid females of $A n$. funestus decreased at temperatures above $28^{\circ} \mathrm{C}$. Overall the numbers of gravid and unfed An. funestus collected in exit collections were not correlated $(p=0.07)$. For an unknown reason the number of $A n$. gambiae s.l. fell below monitoring thresholds during the study. Conclusions: Mean air temperature was the most important environmental parameter 
affecting both vectors in this part of Mozambique. Numbers of An. gambiae s.l. increased at all temperatures recorded whilst $A n$. funestus appeared to be adversely affected by temperatures of $28^{\circ} \mathrm{C}$ and above. These differences may influence the distribution of the vectors as the planet warms. 
1 Some like it hot: A differential response to changing temperatures by the malaria vectors

3

4

5 J.D. Charlwood,

6 Global Health and Tropical Medicine, GHTM, Instituto de Higiene e Medicina Tropical, 7 IHMT, Universidade Nova de Lisboa, UNL, Rua da Junqueira 100, 1349-008 Lisbon, 8 Portugal.

9

12

17 jdcharlwood@gmail.com arabiensis) and Anopheles funestus, respond differently to different temperature regimes.

27 Larval development rates for An. arabiensis peak within the temperature range of $22-32^{\circ} \mathrm{C}$ 
28 with survival rates also highest at $32^{\circ} \mathrm{C}$, whilst an optimal temperature for larval 29 development of An. gambiae is between $28-32^{\circ} \mathrm{C}$ but survival rate to adulthood is highest 30 between $22-26^{\circ} \mathrm{C}$ (Bayoh \& Lindsay, 2003, Christiansen-Jucht et al, 2014). This reflects the 31 higher temperature tolerance of An. arabiensis compared to An. gambiae (Kirby and 32 Lindsay, 2004) which itself may be responsible for the extended distribution of the former 33 species into hotter and drier environments in Africa. Anopheles funestus, on the other hand,

34 has a single optimum temperature of $25^{\circ} \mathrm{C}$ for development with substantial declines in 35 survival either side of this (Christiansen-Jucht, 2014). Le Seur and Sharp (1991) concluded 36 that the effect of temperature on An. merus (another member of the An. gambiae complex) 37 was greatest during metamorphisis in the pupal stage, as did Heuval den val (1963) for 38 Aedes aegypti. Temperature-related metabolism during metamorphosis was affected by 39 available energy reserves (gathered during larval development) (LeSeur and Sharp, 1991). 40 At higher temperatures reserves are lower than at lower temperatures, hence emerging 41 adults are smaller. In the laboratory none of the An. gambiae complex or An funestus 42 survived as larvae or pupae at temperatures above $35^{\circ} \mathrm{C}$. In the field, however, larvae and 43 pupae of An. gambiae s.l. (probably An. arabiensis) have been found in pools at 44 temperatures of $40.5-41.8^{\circ} \mathrm{C}$ (Holstein 1952, quoted in Gillies \& DeMeillon 1968).

The differential response to temperature in the laboratory reflects the different kinds of water in which larval development takes place in the field. Thus, although immature forms of the An. gambiae complex may occur in a great variety of water bodies, the most characteristic are the 'shallow open sun-lit pools with which every field worker in Africa is 50 familiar' (Gillies \& DeMeillon, 1968). Immatures of An. funestus are generally found in 
51 more permanent, shaded, water bodies with emergent vegetation that are cooler than 52 exposed puddles.

53

54 Diurnal fluctuations in temperature also affect the development of many insects 55 (Vangansbeke et al., 2015). In addition to being hotter, temperature fluctuations in small 56 pools are greater than those in larger, shaded, bodies of water. For example, although 57 temperature minima in pools typically used by An. gambiae are similar to the minima in 58 shaded ones, maxima may be $10^{\circ} \mathrm{C}$ higher (Haddow, 1943) and, not surprisingly, given their 59 larval habitat, both An. gambiae and An. arabiensis, also respond better to fluctuating temperatures than do An. funestus (Lyons et al, 2013, Christiansen-Jucht et al., 2014).

61

62 In contrast to the larvae, adults of both An. gambiae s.l. and An. funestus, experience similar 63 microclimates due to their predominantly endophilic behaviour. Temperature influences 64 the time it takes for egg development following a blood-meal but may also have subtler 65 effects. For example, An. funestus delays returning to feed following oviposition at temperatures above $26.5^{\circ} \mathrm{C}$, but at lower temperatures females re-feed shortly after egg

67 laying (Gillies \& Wilkes, 1963). Ironically, the extra time spent in returning to feed at higher temperatures is compensated for by it taking two rather than three days for the mosquito to complete egg development, so that the duration of the complete gonotrophic cycle is three days at all temperatures (Gillies \& Wilkes, 1963).

72 The effect of temperature on the larval stages is manifest in the emergent population. 73 Newly emerged insects can be distinguished from the mature population by their 
74 abdominal and gonotrophic state. Newly emerged males have un-rotated terminalia

75 (Charlwood, 2011) whilst females have undeveloped ovaries and constitute the unfed

76 portion of the resting or exiting population from houses (Gillies and Wilkes, 1965,

77 Charlwood et al., 2003). The relative proportions of engorged to gravid females at different

78 temperatures also provides information on the duration of egg development in mature 79 insects.

80

81 Surprisingly, there remains a lack of comprehensive data on the effects of temperature and 82 other environmental factors on mosquito population dynamics in the wild. Possible effects 83 of temperature on mosquitoes in the field are most easily observed in areas with a wide 84 variation in both daily and seasonal temperatures. Wild mosquito populations are, 85 however, notoriously unpredictable and short-term, chaotic, fluctuations are common. 86 Long-term observations can assist in reducing the 'noise' in such data. Here we describe 87 the effect of ambient temperature, and other environmental parameters, over a five-year 88 period, on the dynamics of newly emerged and mature An. funestus and An. gambiae s.l., 89 collected from a single village in southern Mozambique where temperatures varied from a 90 night-time minimum of $6^{\circ} \mathrm{C}$ in the cool season $\left(10.5^{\circ} \mathrm{C}\right.$ below the lower limit of $16.5^{\circ} \mathrm{C}$ for 91 larval activity, (Jepson et al., 1947)) to a daytime maximum of $35^{\circ} \mathrm{C}$ in the hot season.

92

93

94

95

96

97

\section{Methods}

\section{Description of study site}

The approximately $5 \times 4 \mathrm{~km}$ village of Furvela, (234의 $\mathrm{S}, 35^{\circ} 18^{\prime} \mathrm{E}$ ), $475 \mathrm{~km}$ north of the capital Maputo, is bordered on two sides by the alluvial plain of two river systems (Fig 1). 
99 The Furvela River valley to the north of the village in particular has a considerable amount

100 of local irrigation for agriculture, which provides a large and relatively stable number of

101 small canals. The Inhnanombe river to the east of the village consists largely of beds of the

102 reed (caniço), used for housing, and sugar cane, used in the production of local alcohol; it

103 does not flow as fast as the Furvela river. Anopheles funestus predominates on the Furvela

104 River side of the village and An. gambiae s.l. on the Inhnanombe side (Kampango et al.

105 2013). Malaria is endemic in the village with $80 \%$ of $2-4$ year olds being positive in a cross

106 sectional survey undertaken in 2006 and children under one year of age comprised the

107 majority of attendees at a clinic established by the project in 2001 (supplemental files 1 \& 108 2).

109

110 Houses in the village are generally made with caniço walls and palm thatch roofs. Although

111 most houses don't have windows the majority have a $c a .15 \mathrm{~cm}$ gap between the roof and

112 walls at either end of the house. Doors and doorframes are also generally badly fitting;

113 hence mosquitoes can easily enter these houses. Other styles of house include those with

114 corrugated iron sheets for the roof and those made of concrete blocks (which do have

115 windows). Houses are built either in family compounds of three to six houses or as

116 relatively evenly spaced individual homes.

117 At the start of the study houses were mapped with handheld Global Positioning Units

118 (Garmin etrex), numbered and the manner of construction and size noted (supplemental

119 files $3 \& 4$ ). A census was taken and residents were informed about the purpose of the 120 study and consent concerning the possibility of future mosquito collections obtained. The 
121 initial study area was expanded in the second year of the study and mapping was also

122 repeated in 2007.

123 Mosquito collection

124

125 Light-traps

126

127 Host seeking mosquitoes were collected in CDC light-traps hung, inside bedrooms,

128 approximately $1.5 \mathrm{~m}$ from the floor at the foot of the bed of people who themselves were

129 sleeping under mosquito nets. A random list of houses was produced (using the random

130 number generator in Microsoft Excel) and routine collections were made according to the

131 list. In addition, a number of houses known to have high densities of mosquitoes were used

132 as sentinel sites. Collections were made in 764 houses on the Furvela river side of the

133 village and 214 on the Inhnanombe side of the village. Eleven houses were used for sentinel

134 collections; each being sampled for more than 100 nights.

\section{Exit collection}

136

137 From 2003 to 2007 mosquitoes were also collected exiting houses at dusk (Charlwood,

138 2013). The door of the house was left open and covered with a white mosquito-netting

139 curtain. Mosquitoes were manually aspirated off the curtain as they attempted to leave. See

$140: \underline{\text { https://www.youtube.com/watch?v=SL8FeIuY1GM }}$

141 Most of the houses used for the exit collections were on the Furvela side of the village. The

142 young men and women who did the work, and who lived in dispersed locations collected

143 from their own or nearby houses. Altogether collections were made from 501 houses some

144 of which also acted as sentinel sites.

145 


\section{Resting collection}

147

148 Resting collections, using a torch and an aspirator, were performed on the Furvela side of

149 the village, on an ad hoc basis on 163 days, from a total of 132 houses (mean number of 3.9

150 collections per house) where mosquito nets were not in use, and, initially, outdoors.

151

152

153

154

155

156

157

158

159

160

161

162

163

164

165

166

167

168

169

170

171 Unfortunately, the weather station did not operate throughout the study, nor did the

\section{Mosquito processing}

Collected Anopheles were separated into species or species group, according to the keys of Gillies and De Meillon (1968) and Gillies and Coetzee (1987) and sexed. Females were further separated into unfed, part-fed, engorged, semi-gravid and gravid categories. A number of the An. gambiae s.l. were identified to species by PCR. DNA extraction was performed individually following the protocols of Collins et al. (1988) and the species identified using the protocols of Scott et al. (1993). A small number of An. funestus were also identified by PCR using the protocols of Koekemoer et al. (2002).

\section{Meteorology}

\section{Temperature, insolation and windspeed measurement}

Daily variation estimates of soil and air temperature, insolation and windspeed were obtained with a Delta-T digital weather station (Delta-T Devices, Cambridge, UK) at the edge of the village that recorded hourly information. Soil temperatures approximate those that larvae are exposed to whilst air temperatures are those that more closely approximate those that adults may be exposed to. Unfed females exiting houses at dusk are newly emerged (Charlwood et al., 2011) and reflect the effect of temperature on the larvae whilst the ratio of unfed to gravid insects may reflect temperature effects on the adults. 
172 humidity or rainfall meter work consistently. The longest hourly data sets were from $3^{\text {rd }}$

173 May 2004 to 1 October 2005 and from 10 Nov 2007 to 24 Nov 2008. Hourly data from all

174 years, including the later ones, was amalgamated into daily data and daily data

175 amalgamated into ISO weeks. Mean values for the different ISO weeks from all the weather

176 station files were determined and used in calculations (supplemental file 5)

177

178 Long term temperature data recorded at Vilanculos, a town $\sim 200 \mathrm{~km}$ north of Furvela, 179 were also obtained (long-term data available from www.tutiempo.net).

180

181 Rainfall data

182

183

Rainfall data was available from the town of Maxixe, $20 \mathrm{~km}$ to the south of Furvela. Since

184 the distribution of rainfall is important $(20 \mathrm{~mm}$ falling on seven consecutive days in a week

185 is likely to have a different effect than $140 \mathrm{~mm}$ falling on a single day) a modified measure

186 of rainfall was used to estimate effects:

187

188 Modified weekly rain $=($ Rain $(\mathrm{mm}) * \#$ rain days $) / 7$

189 Thus $140 \mathrm{~mm}$ on a single day is equivalent to $(140 * 1) / 7=20$

190 And $140 \mathrm{~mm}$ with rain every day is equivalent to $(140 * 7) / 7=140$

191 The daily, weekly and monthly records of rainfall over the period 2000-2010 are available

192 at supplemental file 6 (Rainfall data).

193

\section{Analysis}

195

196 Data were entered into, and analyzed with, Excel (supplementary files 6). Unfed 
197 mosquitoes from light-trap collections represent all age groups. Unfed mosquitoes from

198 exit collection are, however, almost entirely newly emerged ones (Charlwood et al., 2011),

199 whilst gravid females have taken at least one blood meal sufficient to develop eggs, and will

200 also include infectious ones. The weekly Williams mean $\left(\log _{10}(n+1)\right)$ of these three groups

201 of An. funestus and An. gambiae s.l. were compared to mean, maximum and minimum

202 temperatures, temperature difference, insolation and wind speed, measured in Furvela, 203 and modified rainfall measured in Maxixe.

204

205 The relationship between mosquito numbers and environmental factors was examined 206 using bivariate correlations, and Pearson's correlation coefficient (2-tailed P value $\leq 0.05$ 207 significance). Least squares multiple linear regression (with climatic factors as 208 independent variables) was also undertaken using the Excel add-in StatPlus. The most 209 parsimonious model was determined by subtraction of least important variables.

213 The study was conducted under the aegis of the joint Instituto Nacional de Saúde (INS)-

214 DBL Centre for Health Research and Development project 'Turning houses into traps for 215 mosquitoes', which obtained ethical clearance from the National Bioethics Committee of 216 Mozambique on 2 April 2001 (ref: 056/CNBS/01). Householders were informed about the 217 purpose of the collections. Verbal consent was obtained when collections were initiated. 
222 Mean temperatures recorded at Vilanculos were higher than those recorded in Furvela, but

223 both followed a similar pattern. (supplemental file 7 Temperature data) There was both a

224 marked seasonality in temperatures and considerable variation from one day to the next.

225 Mean soil and air temperatures from Furvela, derived from hourly measurements, 10 Nov

2262007 to 24 Nov 2008 are shown in Fig 2. Diurnal variation in temperature differed between

227 cool and hot seasons. Figure 3A shows the diurnal pattern recorded on the three coolest

228 nights of the year (16-18 ${ }^{\text {th }}$ July) and 3B the three warmest (14-16 ${ }^{\text {th }}$ October). Overall at the

229 higher temperatures daily variation (difference between maximuim and minimum

230 temperature) was less than it was at the cooler temperatures (Fig 4). At a mean of $23.7^{\circ} \mathrm{C}$

231 the variation in air temperatures was 5.9 degrees and at $18.1^{\circ} \mathrm{C}$ was 15.7 degrees. Variation

232 is soil temperature was lower than air temperatures being 4.1 degrees at $30.3^{\circ} \mathrm{C}$ and 8.9

233 degrees at $28.2^{\circ} \mathrm{C}$.

234

235 Except for the mornings mean soil temperatures were consistently circa $5^{\circ} \mathrm{C}$ warmer than 236 air temperatures. Mean amounts of insolation showed a similar pattern to temperature.

Mosquito data

239

240 Twenty-six (86\%) of 30 males and 331 (81.3\%) of the 407 females from an unselected

241 sample of the An. gambiae complex identified by PCR from 2002 and 2004 comprised $A n$.

242 gambiae, the other species being 4 male and 67 (16.5\%) female An. arabiensis and nine

243 (2.2\%) female An. merus (Table1). There was no statistical difference in the ratio of $A n$.

244 gambiae and An. arabiensis according to method of collection (light-trap, exit collection or 245 resting collection) (supplemental file 8). 
247 All of An. funestus examined morphologically had a single pale spot on the upper branch of 248 the 5th vein and did not have a pale spot at the tip of the 6th vein and corresponded to An. 249 funestus. Seventy-one females of the An. funestus group were identified by PCR 250 (supplementary file 9, courtesy of A. Szlanski). All were An. funestus. Given that this is the 251 endophilic member of the species group, and that it was endophilic behavior that we 252 studied, we assume that this was the only member of the species group present in our 253 collections.

301, 705 female An. funestus were collected in 6043 light-trap collections, 161, 466 in 7397 exit collections and 16, 995 in 1315 resting collections. The equivalent numbers for $A n$. gambiae s.l. are 72, 475 in light-traps, 33, 868 in exit collections and 5, 333 from indoor resting collections (supplemental file 10- Raw data). Outdoor resting collections failed to 259 produce any mosquitoes. Other anopheline species collected in light traps included 5776 Anopheles tenebrosus, 725 Anopheles letabensis, 22 Anopheles rufipes, five Anopheles squamosus, and a single Anopheles pharoensis. A further 219 An. tenebrosus and five An. rufipes were collected exiting houses.

Figure 5 shows the weekly mean numbers collected per house per night of An. funestus and 265 An. gambiae s.l. in light traps and exit collections over the course of the study in conjunction with temperatures recorded at Vilanculos and modified rainfall from Maxixe. Over the three years when both light trapping and exit collections were simultaneously undertaken 268 (2003-2006) mean numbers of An. funestus per house, per method, were similar. In 2007 a 269 cordon sanitaire of long lasting insecticide nets (LLIN's) was established around the 
270 Furvela River valley (Charlwood et al., unpublished) and numbers in exit collections

271 decreased relative to numbers in light-traps. Numbers of An. gambiae s.l. in exit and light-

272 trap were also similar. Over the course of the project, however, An. gambiae s.l.

273 disappeared from both light-trap and exit collection collections. Given the possible effect of

274 the cordon sanitaire on numbers collected further analysis is confined to the years 2001-

2752006 (when 5090 light-trap, 4461 exit and 1315 resting collections were performed).

276

277 Numbers of mosquito were greatest in the warmer months. Figure 6 shows the mean 278 adjusted rainfall from Maxixe, mean soil and air temperature and wind speed from Furvela, 279 mean numbers of unfed An. funestus and An. gambiae s.l. collected in light-traps and mean 280 numbers of unfed and gravid insects by species from exit collections by ISO week in the 281 years 2001-2006. Unfed insects in exit collections comprised the newly emerged portion of 282 the population. Gravid insects represent the gonotrophically active (mature) population. 283 The proportion of that population that they represent depends on the duration of 284 oogenesis following a blood meal. At warmer temperatures development takes two days 285 and so the gravid sample represents half of the mature population whereas at cooler 286 temperatures development may take three days and so the gravid sample will only 287 represent one third of the mature population. The whole of the newly emerged population, 288 on the other hand, is sampled every day.

291 The maximum Pearson correlation co-efficients between mosquito numbers and individual 292 environmental parameters are shown in Table 2. Table 2 also gives the correlation co- 
293 efficients between the different mosquito groups. The highest environmental correlations

294 and mosquito numbers were between temperature and all collections of An. gambiae s.l..

295 (see supplemental file 11 for the other possible correlations). Adding a lag of up to four

296 weeks to the light-trap data from either species did not improve the Pearson correlation

297 co-efficient between rainfall and numbers (Table 3) Correlation co-efficients were always

298 lower in comparisons involving An. funestus.

299

300 Figure 7A gives the relationship between numbers of An. funestus and An. gambiae s.l. 301 caught in light-traps and air temperatures and 7B between mosquito numbers and soil 302 temperature ( $\mathrm{r}^{2}=0.52$ for An. funestus and 0.77 for An. gambiae s.l.). Although both species 303 increased significantly as temperatures increased, numbers of An. gambiae s.l. went 304 through a one hundredfold change (from a mean of 0.14 mosquitoes a night to 14 ) whereas 305 numbers of An. funestus merely doubled (from a mean of 20 to 40 a night).

306

307 The number of unfed and gravid insects in exit collections by mean air temperature are 308 shown in Fig 8A \& 8B. At temperatures below $28^{\circ} \mathrm{C}$ the mean number of gravid An. funestus 309 collected increased as the temperature increased; and at a faster rate than the rate of 310 increase in immature insects but at temperatures above $28^{\circ} \mathrm{C}$ the number decreased whilst 311 numbers of newly emerged insects continued to increase (Fig 8A). Numbers of both gravid 312 and unfed An. gambiae complex females in exit collections continued to increase at all 313 temperatures recorded (Fig 8B). The proportion of the An. gambiae s.l. population that was 314 gravid was more variable at lower temperatures. This was probably due to the small 315 sample sizes at these temperatures. The proportion of An. funestus on the other hand was 
316 more variable at the higher temperatures but why this should be so we do not know since

317 the data comes from a time when large-scale interventions had not been applied. Overall 318 the numbers of gravid and unfed An. funestus collected in exit collections were not 319 correlated $(\mathrm{p}=0.07)$

320

321 The best models for each species and each category of mosquito are given in Table 4. 322 Adjusted rainfall was only included in one model. The models explained more of the 323 variation in An. gambiae s.l. than they did for An. funestus although the environmental 324 parameter used in the models, with the exception of the best model for An. funestus and An. 325 gambiae s.l. in light-traps, were the same. The explanatory values (the adjusted $\mathrm{R}^{2}$ ) were 326 all higher for the An. gambiae s.l. than for the An. funestus.

328 The abdominal status of mature females collected from 1315 resting collections and mean 329 monthly temperature is shown in Figure 9. For both, species, or species group, a higher 330 proportion of semi-gravid and gravid females compared to engorged females were 331 collected during the cooler months of the year (May to August). In other words, oogenesis 332 was taking longer at the lower temperatures.

334 The proportion gravid to engorged An. funestus of $50 \%$ occurred at $25^{\circ} \mathrm{C}$. Thus, at these 335 temperatures, and above it took two days to mature the ovaries and below this three days 336 post-feeding to mature them. The proportion gravid of An. gambiae s.l. from resting 337 catches was always lower than that of the An. funestus and only reached $50 \%$ at the highest 338 temperatures. At mean temperatures of $21.5^{\circ} \mathrm{C} 76 \%$ of the An. funestus collected were 
339 semi-gravid and gravid. This implies that it was taking not just three but four days to

340 complete gonotrophic development.

341

342 Discussion

343

344 With the possible implications of Global Warming, the effect of temperature and other

345 environmental parameters on the dynamics of malaria vectors in Africa is an area of

346 increasing interest. The temperature in Furvela fluctuates between the minimum and

347 optimum temperature for mosquito development, hence over the linear part of the reaction

348 norm. Temperature was the most important environmental parameter, of those measured,

349 determining mosquito numbers in the village. Although a more in depth assessment would

350 probably improve the interpretation of the results, even in the straightforward analysis

351 presented here mean daily temperatures from either air or soil sensors explained $70 \%$ and

$35235 \%$ of the density changes observed in An. gambiae s.l. and An. funestus respectively

353 (Table 2). As expected, given its rapid developmental time, rates of increase were

354 substantially higher in the An. gambiae s.l. compared to the An. funestus. Sporozoite rates

355 were also highest during the warmer months of the year (supplemental file 12). The

356 combined effect on the entomological infection rate (EIR) and malaria morbidity in the

357 village will be examined elsewhere.

358

359 The population dynamics of An. funestus on the peninsula of Linga Linga across the 360 Morrumbene Bay (Fig 1) is determined by rainfall (Charlwood et al., 2013). The absence of 361 a relationship between rainfall and mosquito numbers, particularly the An. gambiae s.l., in 
362 Furvela is surprising. It may be due to the sandy soil in the village which means that rain is

363 rapidly absorbed and the puddles are rare or absent. At the same time breeding conditions

364 for the An. funestus in the valley remain reasonably constant due to the irrigation practices

365 of the local farmers and even major storms have only a temporary effect on the dynamics of

366 the mosquito (Charlwood and Bragança, 2012).

367

368 The ratio of gravid to unfed mosquitoes in exit collections depends on a number of factors,

369 in particular house construction. The two sets of females enter the house at different times

370 (unfed newly emerged insects entering at dawn to rest and, soon-to-be-gravid, host seeking

371 females, to feed throughout the night). They use different cues (visual contrast and odour)

372 and enter through different routes (open doors and eaves) (Gillies, 1988). Thus, houses

373 that may allow access for one group are not necessarily suitable for the other. In addition

374 to house effects, the proportion of egg development time spent inside houses (which we

375 presume to be $100 \%$, at least for An. funestus), the survival rate per oviposition cycle and

376 the duration of oogenesis, can all affect the unfed/gravid ratio. Should any of these factors

377 change with temperature then the overall ratio will also change with temperature. The

378 absence of change, as occurred with the An. gambiae s.l. implies that these factors remained

379 constant, or compensated exactly, over the observed range of temperatures.

380

381 Ironically, the highest correlation between gravid insects in exit collections (for both 382 species or species group) was with soil temperature (which mimics water temperature) 383 whilst for unfed (newly emerged) insects it was with outdoor air temperature. As pointed 384 out by Pajimans et al., (2010) the micro-climate experienced by the mosquito inside houses 385 may be quite different to that outside. Houses may be warmer in the cool season and 
386 cooler in the hot season than temperatures recorded outside. Nevertheless, more

387 sophisticated measurement would only improve models for An. gambiae by a maximum of $38830 \%$ and for An. funestus by $47 \%$ (and would imply that other factors, such as humidity, 389 were of lesser importance).

390

391 Unlike An. funestus both newly emerged and gravid An. gambiae s.l. increased in a similar 392 fashion through the whole range of temperatures experienced in Furvela. There was no 393 apparent effect of increasing temperatures on survival and the proportion of gravid to 394 unfed insects remained more or less constant at all temperatures. The unfed/gravid ratio 395 of the more common An. funestus did, however, change with temperature. As temperatures 396 increase above $26.5^{\circ} \mathrm{C}$ a higher proportion of gravid $A n$. funestus is to be expected in exit 397 collections since the duration of oogenesis is reduced from three to two days (Gillies and 398 DeMeillon, 1968). At the temperatures recorded in July it might take three or more days, as 399 evidenced in the resting collections and formerly described by Gillies and Wilkes (1963, 400 1965). At the higher temperatures exit collections would therefore be expected to sample 401 one half of the mature population (the other half being the semi-gravid insects that may 402 move from one resting site to another, but in the absence of disturbance, do not leave the 403 house) but at cooler temperatures only one third, or even less, of the population. At the 404 higher temperatures, however, the proportion of gravid insects in the exit collections 405 decreased, such that overall there was no significant relationship between the numbers of 406 gravid and unfed insects in exit collections. This either means that that survival between 407 emergence and maturity (i.e. becoming gravid) decreases at cooler temperatures or that 408 post-teneral insects have a higher mortality at higher temperatures. Both sets of insects 
409 leave houses at the same time (Charlwood, 2011), hence sampling efficiency for the two 410 groups should be the same.

411

412 A reduced survival among post-teneral adult An. funestus at the higher temperatures is 413 possible as described from the laboratory (Christiansen-Jucht et al., 2015). High 414 temperatures, independent of humidity, can have a lethal effect because as body 415 temperature increases, metabolism and respiration increase up to a critical thermal limit, 416 and there is a loss of integration between protein stability and metabolic processes that 417 leads to death. Anopheles gambiae s.l. are larger than An. funestus. Volume to surface ratios 418 differ and this may influence the ability of the adult insects to survive higher temperatures. 419 Larger mosquitoes have a smaller surface to volume ratio and larger water reserves, which 420 would allow them to offset the respiratory and cuticular water loss.

421

422 With one exception, windspeed was the only environmental parameter, other than 423 temperature, included any model. Together they explained up to $82 \%$ of the An. gambiae 424 changes and $61 \%$ of the An. funestus. The exception was An. funestus in light traps in which 425 adjusted rain was included. Although still significant, this model had the lowest explanatory 426 value (34\%) of all the models.

427

428 Recently Pajimanns et al., (2010) have described that in addition to mean temperatures 429 'the key mosquito-related traits that combine to determine malaria transmission intensity 430 (i.e., parasite infection, parasite growth and development, immature mosquito 431 development and survival, length of the gonotrophic cycle, and adult survival) are all 
432 sensitive to daily variation in temperature'. Fluctuations in temperature (i.e. the difference

433 between maximum and minimum temperatures) were greatest in the cooler months. In the

434 cool season the observed patterns in soil and air temperature were similar to shaded and

435 open water as determined by Haddow (1943). In the warmer months' fluctuations in

436 temperature were less than at lower temperatures. Fluctuations around low mean

437 temperatures can speed up rate processes, whereas fluctuations around high mean

438 temperatures can slow them down (Paajimans et al, 2010). Thus, the An. gambiae s.l. were

439 well suited to the temperature regimes experienced in Furvela.

440

441 Unfortunately, we do not know the species composition of the resting or exiting $A n$.

442 gambiae s.l. compared to those entering the house. Nevertheless, the proportion of gravid

443 insects in resting catches varied in a similar fashion to that seen among the An. funestus.

444 We also do not know if the different members of the An. gambiae complex behaved 445 differently or disappeared from the study area at different rates. Nevertheless, all members 446 of the complex did apparently disappear during the study so that perhaps it was not just a 447 specific species that was affected but was a complex wide problem. Meyrowitsch et al., 448 (2011) were unable to determine the cause of the decline of An. gambiae s.l. in the Tanga 449 region of Tanzania, three thousand kilometers to the north of Furvela. In the Kilifi area of 450 Kenya population decline of An. gambiae, shown by a reduction in genetic diversity in the 451 mosquito, was attributed to the introduction of LLIN's (O'Loughlin et al, 2016) The decline 452 in Furvela started before the introduction of any control measures and although the 453 introduction of LLIN's may have exacerbated the problem for the mosquito it may not have 454 been the cause of the decline in the first place. The decline also paralleled that observed in 
455 malaria in the Rufiji basin (Ishengoma et al., 2013). That similar declines appeared to occur

456 over a 3,000 kilometer stretch of coastline indicates that a climatic factor was responsible.

\section{Conclusion}

459 Temperature is a major driving force in the dynamics of both An. funestus and An. gambiae

460 s.l. in Furvela. Whilst gravid An. funestus appeared to be negatively affected by the highest

461 temperatures encountered increasing temperatures were favorable for the An. gambiae s.l.

462 which went through hundredfold changes in densities as a result. As average temperatures

463 increase due to Global Warming areas where An. gambiae is the vector, if there remains

464 sufficient water available for larval development, will be at heightened risk of malaria

465 transmission.

466

467

468

469

470

471

472

473

474

475

476

477

478

479

480

481

\section{Acknowledgements}

I would like to thank Joao Pinto of the IHMT, Portugal for identifying the members of the An. gambiae complex in this study and for his perceptive comments on the manuscript. I also thank Corey LeClair of the LSHTM UK and Olivier Briët of the Swiss TPH for comments on the manuscript. Thanks too to the reviewers of the manuscript whose careful comments improved the manuscript. Thanks to Nelson Cuamba and Ricardo Thompson for logistic

support during the study and thanks to the members of the MOZDAN project and to the villagers of Furvela who let us collect mosquitoes from their houses

\section{References}

Bayoh MN, Lindsay SW. 2003. Effect of temperature on the development of the aquatic stages of Anopheles gambiae sensu stricto (Diptera: Culicidae). Bulletin of Entomological Research. 93:375-381. 
482 Beck-Johnson L, Nelson W, Paaijmans K, Read A, Thomas M, Bjørnstad 0. 2013. The 483 effect of temperature on anopheles mosquito population dynamics and the potential for 484 malaria transmission. PLoS One 8(11):e79276.

485

Charlwood JD. 2011. Studies on the biology of male Anopheles gambiae Giles and Anopheles funestus Giles from southern Mozambique. Journal of Vector Ecology 36: 382-394.

488

Charlwood JD, Braganca M. 2012. The effect of rainstorms on adult Anopheles funestus behavior and survival. Journal of Vector Ecology 2012a 37: 1-5

491

Charlwood JD, Cuamba N, Tomás EVE. Briët OJT. 2013. Living on the edge: a longitudinal study of Anopheles funestus in an isolated area of Mozambique. Malaria Journal 12:208

495

Charlwood JD, Thompson R, Madsen H. 2003. Swarming and mating in Anopheles 497 funestus from southern Mozambique. Malaria Journal 2003, 2:3

498

499

500

501

502

503

504

505

506

507

508

509

510

511

512

513

514

515

516

517

518

519

520

521

522

Christiansen-Jucht CD, Parham PE, Saddler A, Koella JC, Basáñez M-G. 2014 Temperature during larval development and adult maintenance influences the survival of Anopheles gambiae s.s. Parasites \& Vectors. 2014; 7:489. doi:10.1186/s13071-014-0489-3.

Christiansen-Jucht CD, Parham PE, Saddler A, Koella JC, Basáñez M-G. 2015. Larval and adult environmental temperatures influence the adult reproductive traits of Anopheles gambiae s.s. Parasites \& Vectors. 2015; 8:456. doi:10.1186/s13071-015-1053-5.

\section{Collins FH, Petrarca V, Mpofu S, Brandling-Bennett AD, Were JB, Rasmussen MO,}

Finnerty V. 1988. Comparison of DNA probe and cytogenetic methods for identifying field collected Anopheles gambiae complex mosquitoes. American Journal of Tropical Medicine \& Hygiene 39, 545-550.

Gillies MT. 1988. Anopheline mosquitoes: vector behavior and bionomics. Chapter 16 In WH Wersdorfer \& I McGregor (eds) Malaria, Principles and Practice of Malariology. Churchill Livingstone, Edinburgh

Gillies MT, Coetzee M. 1987. A Supplement to the Anophelinae of Africa South of the Sahara (Afrotropical Region)Publication no. 55. South African Institute for Medical Research, Johannesburg.

Gillies MT, De Mellion B. 1968. The Anophelinae of Africa South of the Sahara (Ethiopian Zoogeographical Region), 2ndPublication no. 54 edn. South African Institute for Medical Research, Johannesburg. 
523 Gillies MT, Wilkes TJ. 1963. Observations on nulliparous and parous rates in a 524 population of Anopheles funestus in East Africa. Annals of Tropical Medicine and 525 Parasitology. 57: 204-213.

526

527

528

529

530

531

532

533

534

535

536

537

538

539

540

541

542

543

544

545

546

547

548

549

550

551

552

553

554

555

556

557

558

559

560

561

562

563

564

565

566

Gillies MT, Wilkes TJ. 1965. A study of age composition of population of Anopheles gambiae Giles and Anopheles funestus Giles in North-eastern Tanzania. Bulletin of Entomological Research 56: 129-135.

Haddow AJ. 1943. Measurements of temperature and light in artificial ponds with reference to the larval habitat of Anopheles (Myzomyia) gambiae Giles, and A. (M.) funestus Giles. Bulletin of Entomological Research 34:89-93.

Heuval MJ van den. 1963. The effect of rearing temperature on the wing length, thorax length, leg length and ovariole number of the adult mosquito, Aedes acgyptii (L.). Transactions of the Royal Entomological Society of London, 115, 197-216.

Holstein M. 1952. Biologie d'Anopheles gambiae. Monograph Series World Health Organization No 9, 176 pp.

Ishengoma DA, Mmbando BP, Segeja MD, Alifrangis M, Lemnge MM, Bygbjerg IC. 2013. Declining burden of malaria over two decades in a rural community of Muheza District, north-eastern Tanzania. Malaria Journal 12:338 DOI 10.1186/1475-2875-12-338.

Jepson WF, Moutia A, Courtis C. 1947. The malaria problem in Mauritius: the bionomics of Mauritian anophelines. Bulletin of Entomological Research, 38:177-208.

Kampango A, Bragança M, de Sousa B, Charlwood JD. 2013. Netting barriers to prevent mosquito entry into houses in southern Mozambique: a pilot study. Malaria Journal 12:99.

Koekemoer LL, Kamau L, Hunt RH, Coetzee M. 2002. A cocktail polymerase reaction assay to identify members of the Anopheles funestus (Diptera: Culicidae) group. American Journal of Tropical Medicine and Hygiene, 66, 804-811.

Kirby MJ, Lindsay SW. 2004. Responses of adult mosquitoes of two sibling species, Anopheles arabiensis and An. gambiae s.s. (Diptera: Culicidae), to high temperatures. Bulletin of Entomological Research. 94:441-448.

Le Sueur, Sharp BL. 1991. Temperature-dependent variation in Anopheles merus larval head capsule width and adult wing length: implications for anopheline taxonomy. Medical and Veterinary Entomology 5: 55-62.

Lyons CL, Coetzee M, Chown SL. 2013. Stable and fluctuating temperature effects on the development rate and survival of two malaria vectors, Anopheles arabiensis and Anopheles funestus. Parasites \& Vectors. 6:104. doi:10.1186/1756-3305-6-104. 
567 Meyrowitsch DW, Pedersen EM, Alifrangis M, Scheike TH, Malecela MN, Magesa SM, 568 Derua YA, Rwegoshora RT, Michael E, Simonsen PE. 2011. Is the current decline in 569 malaria burden in sub-Saharan Africa due to a decrease in vector population? Malaria 570 Journal, 10:188

571 O'Loughlin SM, Magesa SM, Mbogo C. Mosha F. Midega J. Burt A. 2016. Genomic 572 signatures of population decline in the malaria mosquito Anopheles gambiae Malaria 573 Journal 15:182 DOI: 10.1186/s12936-016-1214-9

574 Paaijmans KP, Blanford S, Bell AS, Blanford JI, Read AF, Thomas MB. 2010. Influence of 575 climate on malaria transmission depends on daily temperature variation. Proceedings of the 576 National Academy of Science USA 107: 15135-9.

Scott JA, BrogdonWG, Collins FH. 1993. Identification of single specimens of the

580 Medicine and Hygiene, 49, 520-529.

581 


\section{Table $\mathbf{1}$ (on next page)}

PCR identifications of members of the An. gambiae complex collected from light-traps, Furvela Village, Mozambique. 
1

\begin{tabular}{lcccccccc}
\hline Year & 2002 & & 2003 & & 2004 & \multicolumn{3}{c}{ Total } \\
Species & $N$ & $\%$ & $N$ & $\%$ & $N$ & $\%$ & $N$ & $\%$ \\
\hline \hline A. arabiensis & 16 & 27.1 & 35 & 23.3 & 20 & 8.8 & 71 & 16.2 \\
A. gambiae & 43 & 72.9 & 108 & 72.0 & 206 & 90.4 & 357 & 81.7 \\
A. merus & 0 & 0.0 & 7 & 4.7 & 2 & 0.9 & 9 & 2.1 \\
Total per year & 59 & 100.0 & 150 & 100.0 & 228 & 100.0 & 437 & 100.0 \\
\hline
\end{tabular}

2

3

5 


\section{Table 2 (on next page)}

Correlation coefficients between mosquitoes and climate

Correlation coefficients between weekly mean numbers of mosquitoes according to the collection method and the highest correlation co-efficient by environmental variable, Furvela, Mozambique 2003-2006. 
1

2

3

\begin{tabular}{|c|c|c|c|c|}
\hline Species & Sample & $\begin{array}{c}\text { Environmental } \\
\text { Variable }\end{array}$ & $\begin{array}{l}\text { Correlation } \\
\text { co-efficient }\end{array}$ & $p$ \\
\hline \multirow{4}{*}{$\begin{array}{l}\text { Anopheles } \\
\text { funestus }\end{array}$} & Light-trap & Air Temperature & 0.5352 & $>0.0005$ \\
\hline & Unfed Exit & Min Air Temperature & 0.71959 & $>0.0005$ \\
\hline & Gravid Exit & Max Solar & 0.68915 & $>0.0005$ \\
\hline & Unfed Exit & Gravid Exit & 0.25169 & 0.071 \\
\hline \multirow{4}{*}{$\begin{array}{l}\text { Anopheles } \\
\text { gambiae s.l. }\end{array}$} & Light-trap & Air Temperature & 0.75105 & $>0.0005$ \\
\hline & Unfed Exit & Air Temperature & 0.73002 & $>0.0005$ \\
\hline & Gravid Exit & Air Temperature & 0.74211 & $>0.0005$ \\
\hline & Unfed Exit & Gravid Exit & 0.86102 & $>0.0005$ \\
\hline \multirow[t]{2}{*}{ funestus/gambiae } & Unfed Exit & Unfed Exit & 0.58979 & $>0.0005$ \\
\hline & Gravid Exit & Gravid Exit & 0.44756 & 0.0009 \\
\hline
\end{tabular}




\section{Table 3(on next page)}

Correlation between mosquitoes and rainfall

Pearson correlation co-efficients between adjusted rainfall and numbers of An. gambiae s.l. and $A n$. funestus collected in light traps at lags of $0-4$ weeks. 
1
2
3

\begin{tabular}{ccc}
\hline Lag (weeks) & An. gambiae s.l. & An. funestus \\
\hline 0 & 0.671 & 0.245 \\
1 & 0.435 & 0.285 \\
2 & 0.517 & 0.275 \\
4 & 0.358 & 0.150 \\
4 & 0.379 & 0.248 \\
\hline
\end{tabular}

4

5 


\section{Table 4(on next page)}

Models and mosquito numbers

Environmental regression models for the different categories of female mosquito collected, Furvela, 2001-1007 
1

2

\begin{tabular}{|c|c|c|c|c|}
\hline Species & Category & Parameters & $\mathrm{R}^{2}$ & $\mathrm{p}$ \\
\hline \multirow[t]{3}{*}{ An. funestus } & Unfed exit & $\begin{array}{l}\text { Air temp + } \\
\text { windspeed }\end{array}$ & 0.611 & $>0.00005$ \\
\hline & Gravid exit & $\begin{array}{l}\text { Soil temp + } \\
\text { windspeed }\end{array}$ & 0.563 & $>0.00005$ \\
\hline & Unfed Light & $\begin{array}{l}\text { Air temp + } \\
\text { Rain }\end{array}$ & 0.344 & $>0.00005$ \\
\hline \multirow[t]{3}{*}{$\begin{array}{l}\text { An. gambiae } \\
\text { s.l. }\end{array}$} & Unfed exit & $\begin{array}{l}\text { Air temp + } \\
\text { Windspeed }\end{array}$ & 0.752 & $>0.00005$ \\
\hline & Gravid exit & $\begin{array}{l}\text { Soil temp + } \\
\text { Windspeed }\end{array}$ & 0.822 & $>0.00005$ \\
\hline & Unfed Light & $\begin{array}{l}\text { Air temp + } \\
\text { Windspeed }\end{array}$ & 0.756 & $>0.00005$ \\
\hline
\end{tabular}

The equations for the different models are listed below:

Log An. funestus Light-trap $=+0.9178-0.445^{*} \log$ rain $+0.0342^{*}$ Air temp

Log An. gambiae s.l. Light-trap $=-1.419+0.117^{*}$ Air temp $-0.392^{*}$ Windspeed

Exit An. funesus unfed $=-0.100+0.044^{*}$ Air temp $+0.156^{*}$ Windspeed

Exit An. funestus gravid $=-0.099+0.0456 *$ Soil temp $-0.111^{*}$ Windspeed

Exit An. gambiae s.l. unfed $=-1.629+0.1097 *$ Air temp $-0.446 *$ Windspeed

Exit An. gambiae s.l. gravid $=-2.05+0.10869$ Soil temp $-0.734 *$ Windspeed 


\section{Figure 1 (on next page)}

Google Earth image of Furvela Village

Furvela village and its relation to Linga Linga. 


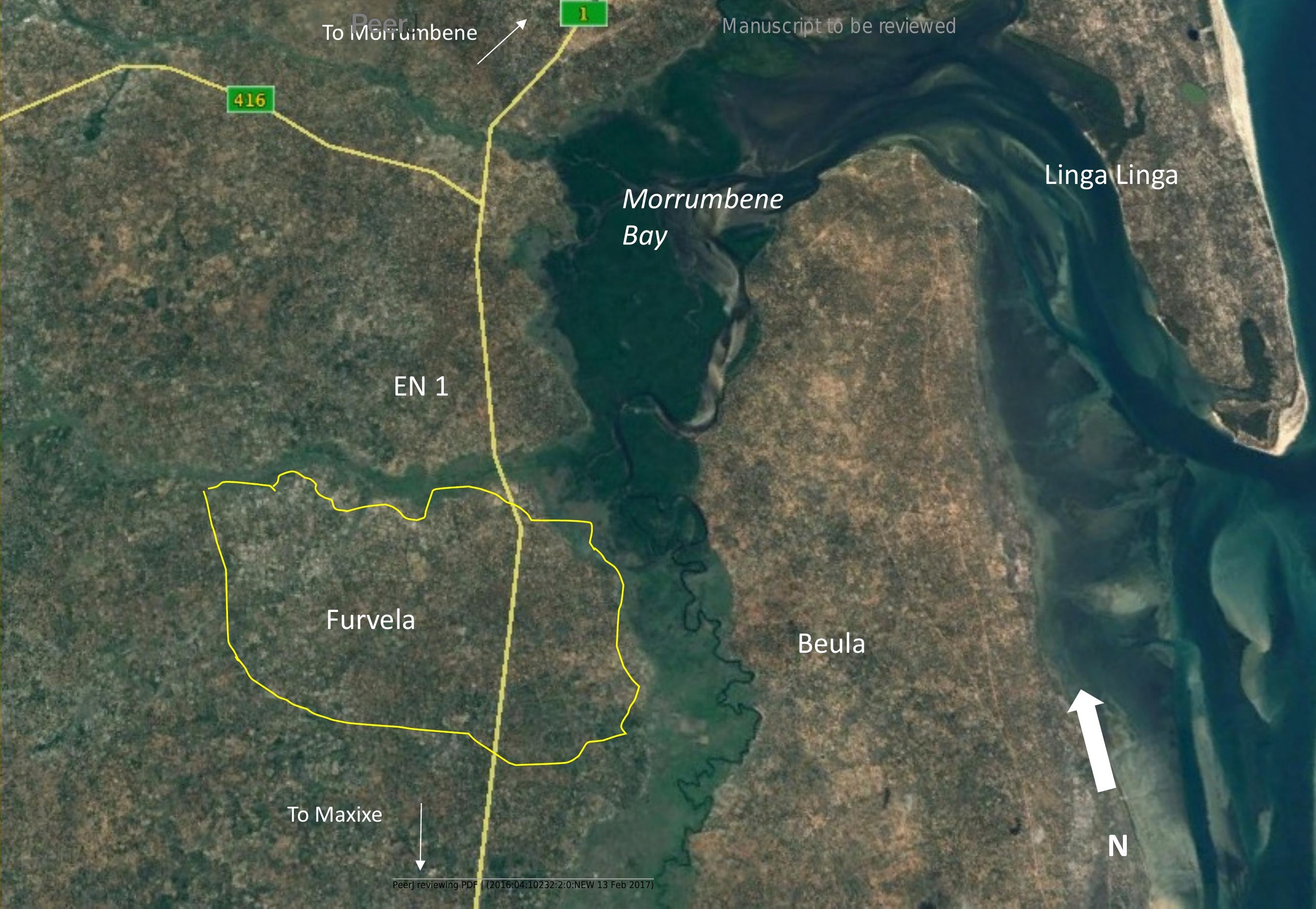


Figure 2 (on next page)

Annual temperature variation

Mean daily soil and air temperatures recorded by the Delta logger in Furvela village 


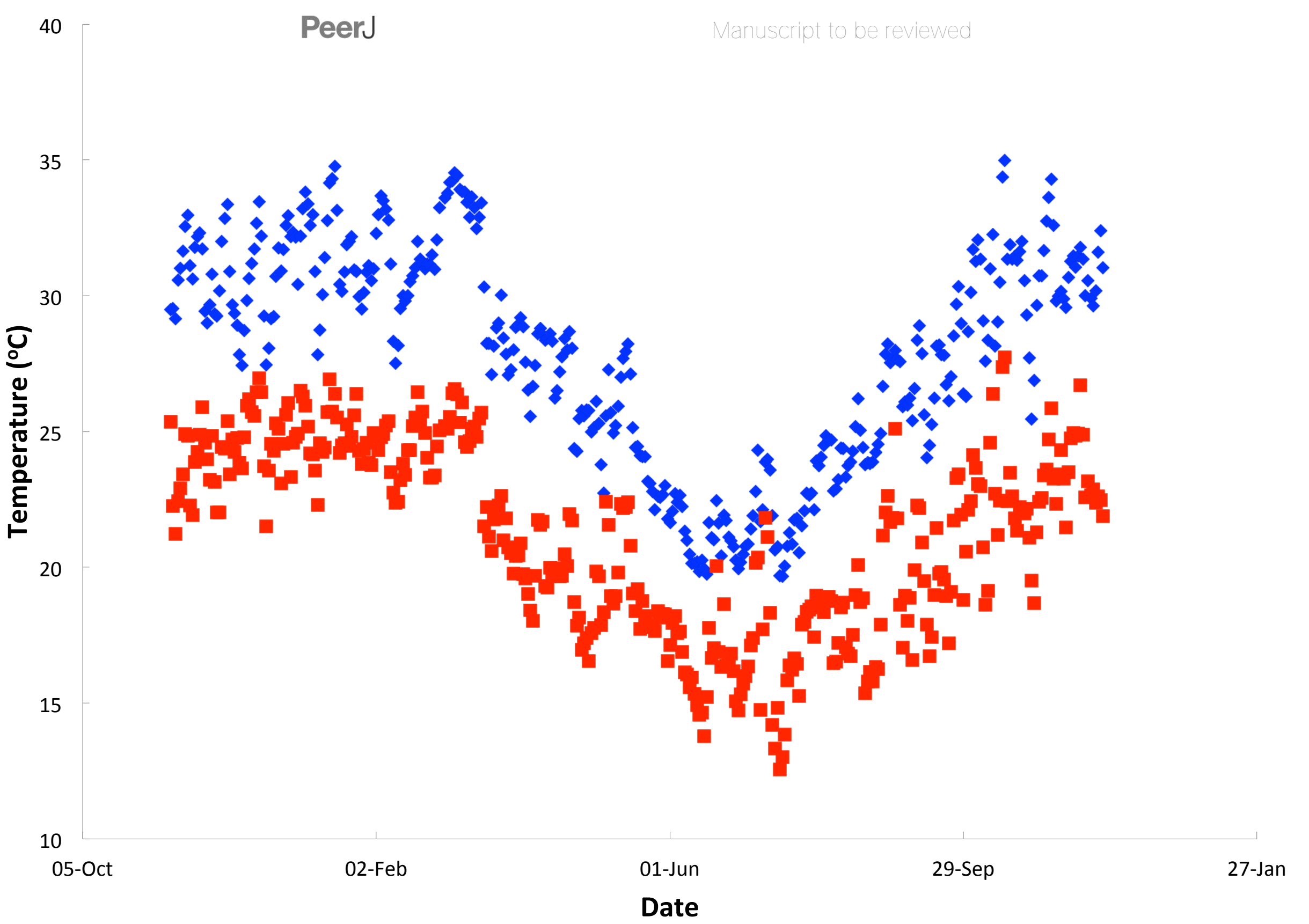




\section{Figure 3 (on next page)}

Daily variation in temperature during A) the coolest months of the year and B) the warmest months of the year, Furvela, Mozambique. 
Figure 4 (on next page)

Difference between maximum and minimum air and soil temperature recorded from Furvela village, Mozambique, by ISO week number. 


\section{Figure 5 (on next page)}

Temperature, rainfall and number of mosquitoes collected in Furvela

Upper graph -Rainfall (measured in Maxixe) (Blue histogram), air temperature (measured in Vilanculos) (orange line)

(A) mean numbers of unfed Anopheles funestus form light-traps (blue line) and in exit collections (green line)

(B) mean numbers of unfed Anopheles gambiae collected from light-traps (Dark blue line) and in exit collections (light blue line) from Furvela village, 2001-2009. Note the log scale. 


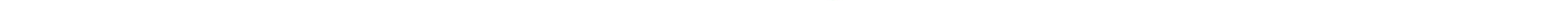




\section{Figure 6 (on next page)}

Weekly mean adjusted rain, soil temperature, air temperature, windspeed and mosquito numbers

Environmental parameters and mosquito numbers by ISO week, Furvela, Mozambique. A) mean weekly adjusted rain in millimetres (measured in Maxixe), B) Light blue line - mean soil temperature (in degrees Centigrade); Black solid line Mean air temperature (in degrees centigrade); dotted line - mean wind-speed (in metres per second),C) Mean number of Anopheles funestus (red line) and An. gambiae s.I. (blue line) collected from light traps, D) Mean number of unfed (pink line) and gravid (red line) An. funestus from exit collections,(note the log scale) E) Mean number of unfed (light blue line) and gravid (dark blue line) An. gambiae s.l. from exit collections, (note the log scale). 

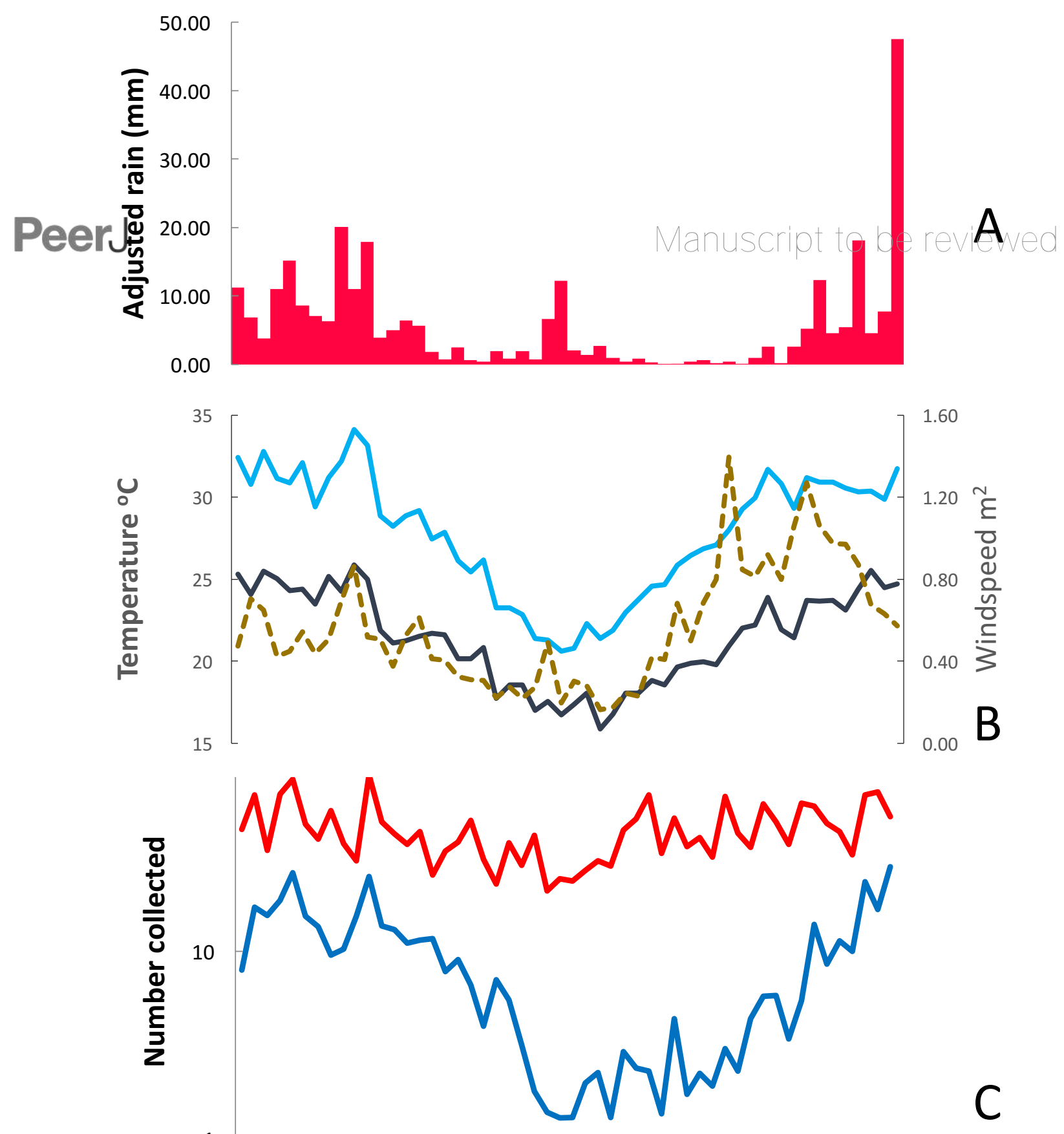

C

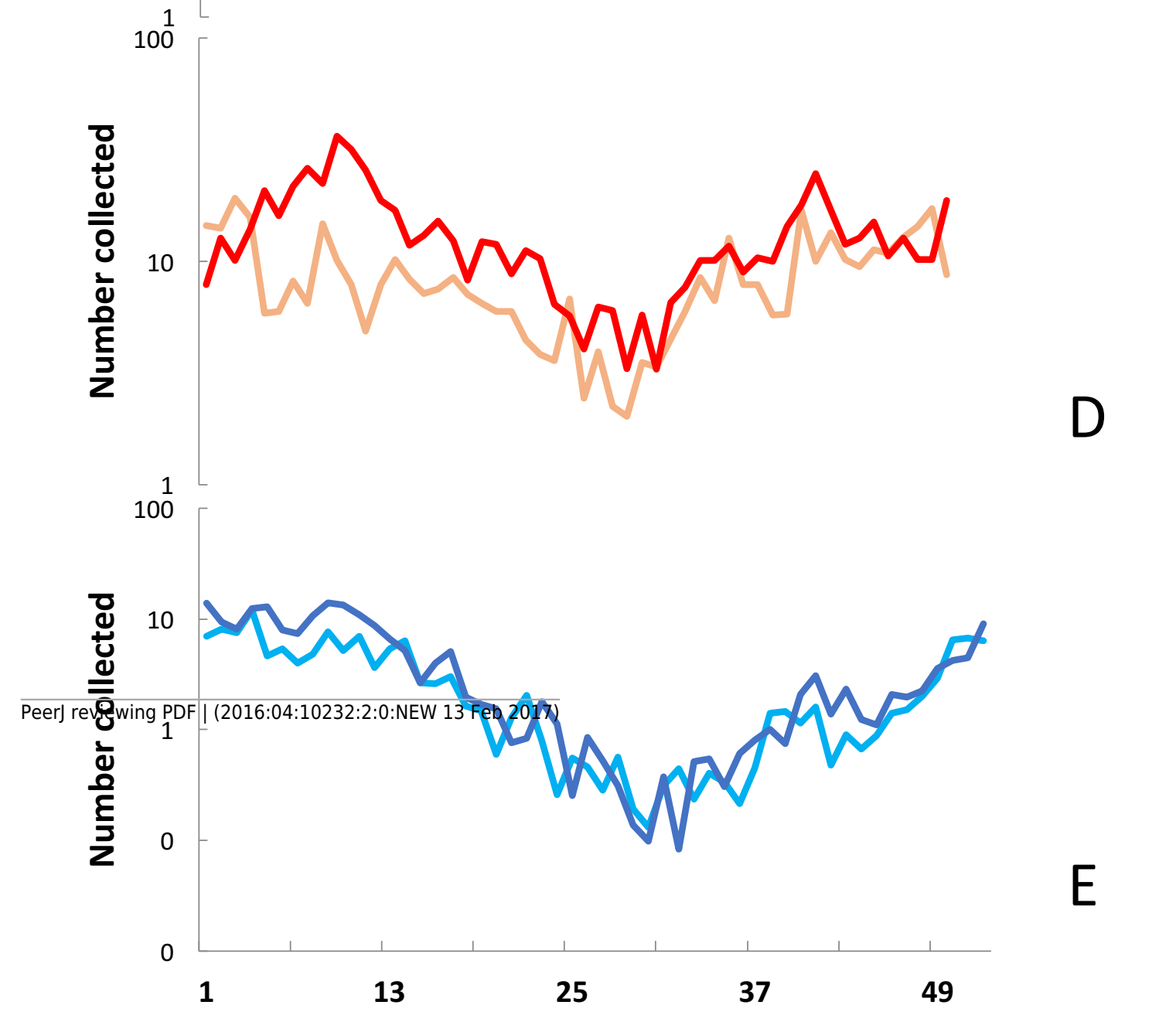

Week number 
Figure 7 (on next page)

Mean numbers of Anopheles funestus (red) and An. gambiae s.l. (blue) collected in lighttraps from Furvela village, Mozambique $A$ ) by air temperature and $B$ ) by soil temperature (in degrees Centigrade) 

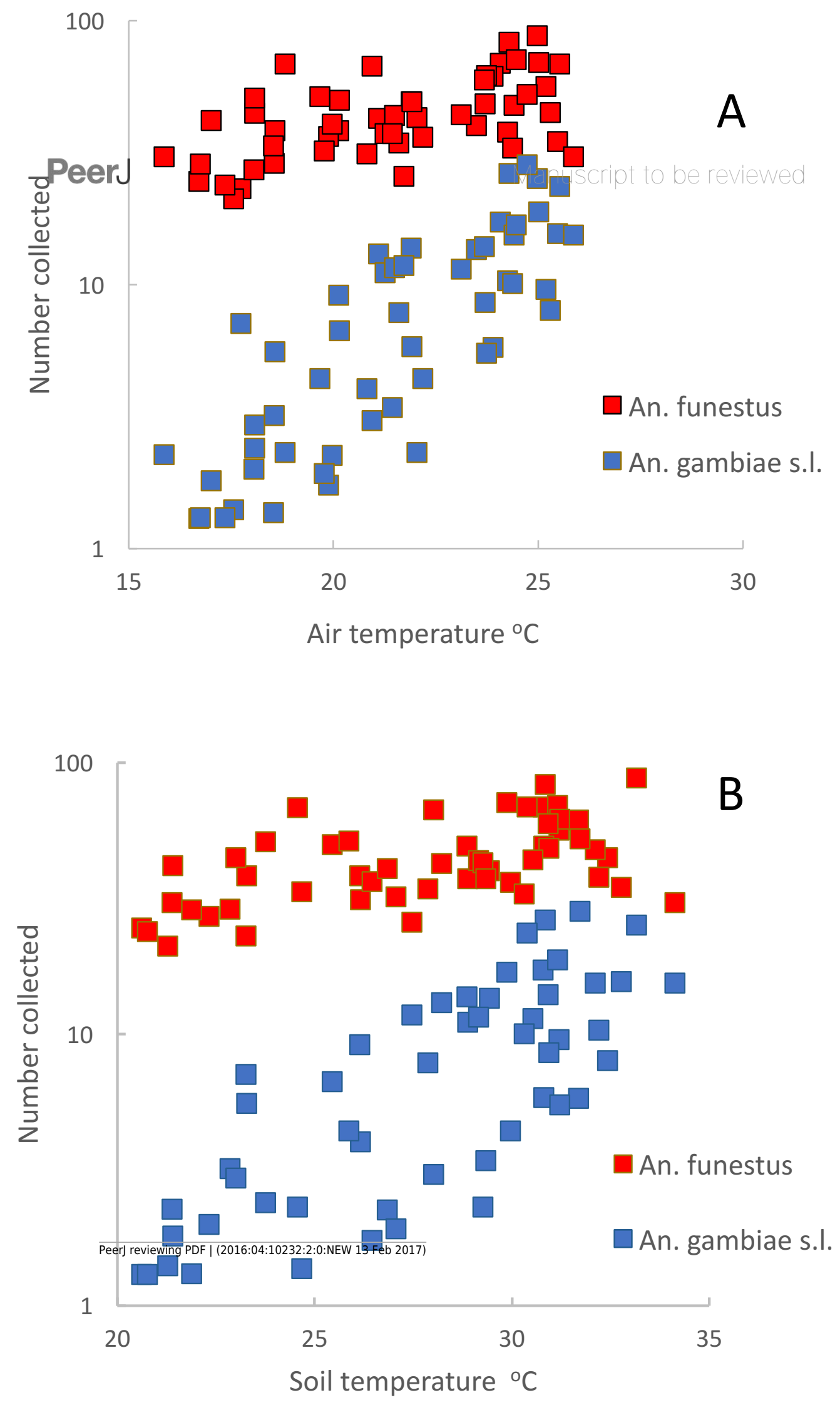


\section{Figure 8 (on next page)}

The relationship between the mean number of A) Anopheles funestus and B) An. gambiae s.l. in exit collections and soil temperature Furvela, Mozambique. 


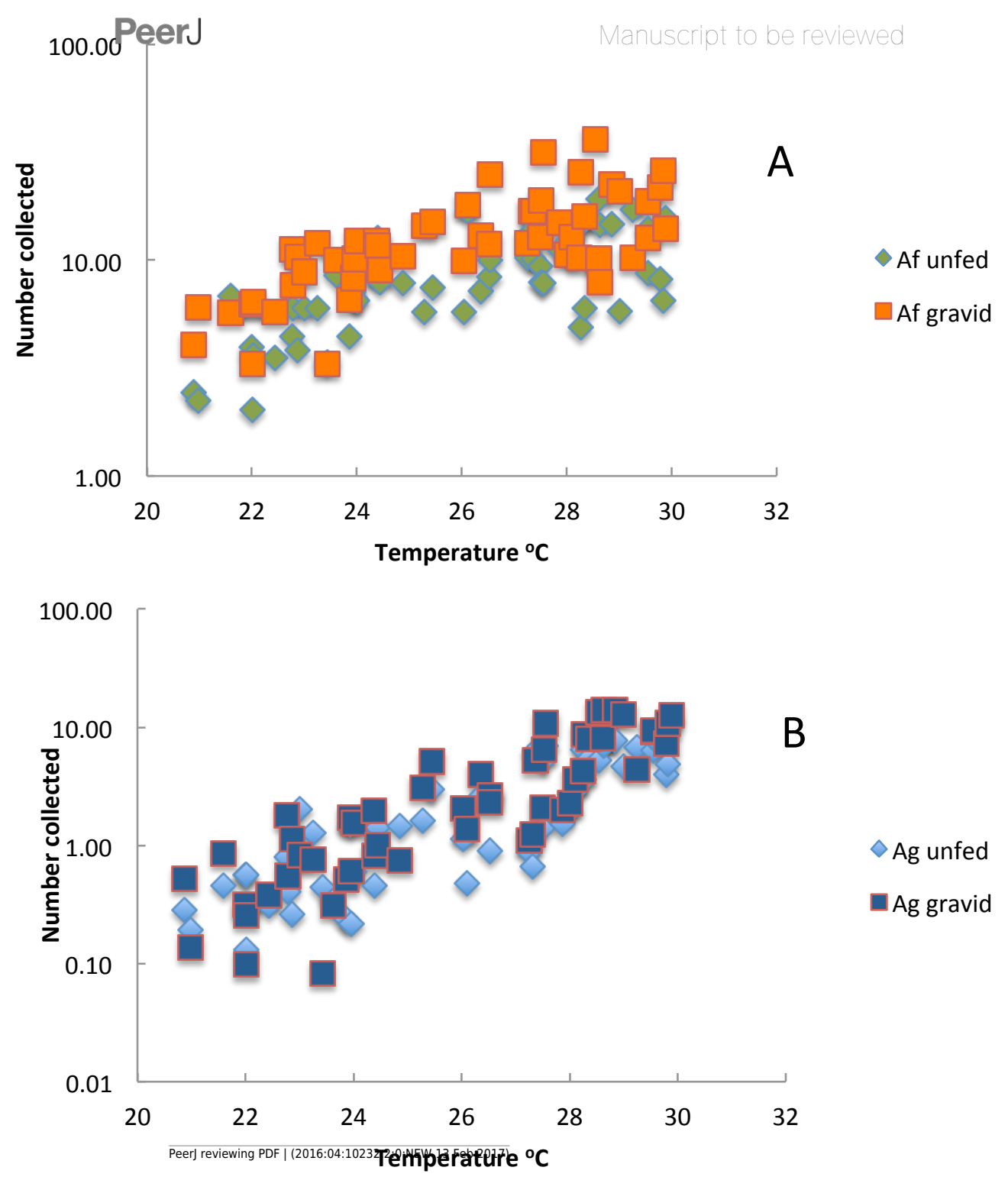


Figure 9 (on next page)

Proportion of Anopheles funestus and An. gambiae s.l. indoor resting that were gravid at the time of collection and mean temperature, Furvela Mozambique.

The proportion of female Anopheles funestus and An. gambiae s.l. indoor resting that were gravid at the time of collection and mean air temperatur, Furvela, Mozambique. 


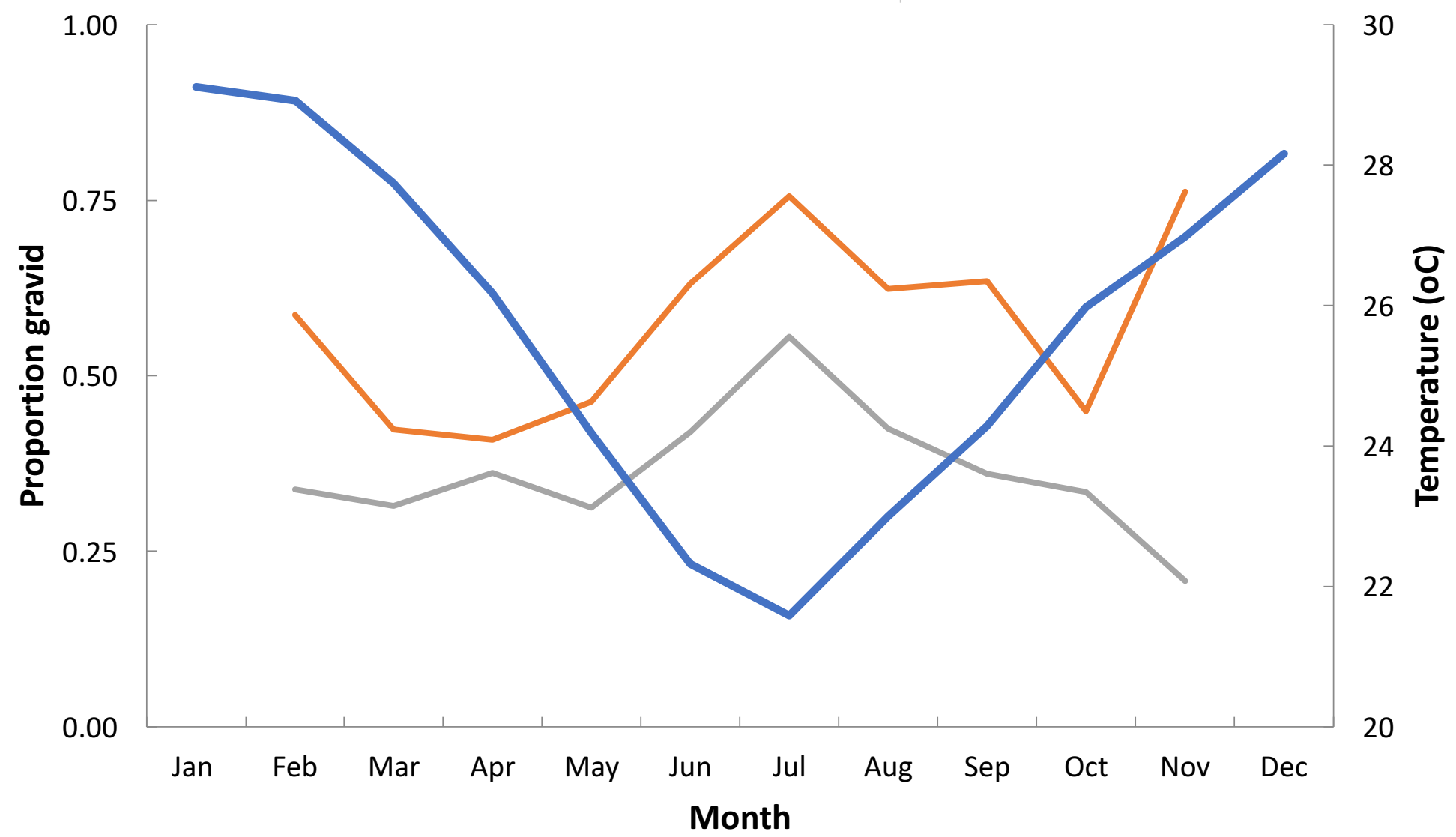

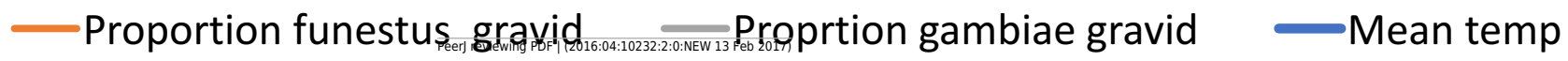

